
\title{
25 Research Square \\ Risk Factors for Mortality of Patients with COVID-19 in Korea: A Nationwide Population-Based Study
}

\section{Se Jun Park}

NHIC Ilsan Hospital: National Health Insurance Corporation Ilsan Hospital

\section{Hyungkook Lee}

NHIC Ilsan Hospital: National Health Insurance Corporation Ilsan Hospital

\section{Tae Mi Youk}

NHIC Ilsan Hospital: National Health Insurance Corporation Ilsan Hospital

Han Ho Jeon ( $\nabla$ fortune22c@nhimc.or.kr)

NHIC Ilsan Hospital: National Health Insurance Corporation Ilsan Hospital https://orcid.org/0000-00023393-3304

\section{Research Article}

Keywords: Coronavirus disease 2019, Mortality risk, Comorbidities

Posted Date: October 8th, 2020

DOI: https://doi.org/10.21203/rs.3.rs-86517/v1

License: (c) (1) This work is licensed under a Creative Commons Attribution 4.0 International License. Read Full License 


\section{Abstract}

\section{Background}

Coronavirus Disease 2019 (COVID-19) is an ongoing major global health crisis caused by severe acute respiratory syndrome coronavirus 2 (SARS-CoV-2). The outcomes of COVID-19 in patients remain unclear. We aimed to identify risk factors for the mortality of COVID-19 in the Republic of Korea without health system disruption.

\section{Methods}

The data of confirmed COVID-19 patients were retrieved from Health Insurance Review and Assessment Service (HIRA) dataset between January 3 and June 1, 2020. We used multivariable logistic regression models to identify risk factors for mortality in patients with COVID-19.

\section{Results}

We identified 7,333 COVID-19 patients, of whom 227 (3.1\%) died. Hypertension was the most common (26\%), followed by diabetes mellitus (23.0\%). In multivariate analysis, male (OR: $1.92, \mathrm{P}<0.0001)$, elderly (age >65) (OR: 11.00, $P<0.0001$ ), living in a large outbreak area (OR: 2.61, $P<0.0001$ ), Medical Aid beneficiaries (OR: 1.54, $P=0.0282$ ), Charlson Comorbidity Index (CCl) score $\geq 3$ (OR: 3.25, $P=0.029$ ), hypertension (OR: 1.90, $\mathrm{P}=0.0013)$, chronic obstructive pulmonary disease (COPD) (OR: 1.61, $\mathrm{P}=$ 0.0374), congestive heart failure (CHF) (OR: 2.14, $\mathrm{P}=0.0001)$, and end-stage renal disease (ESRD) (OR: $5.69, \mathrm{P}=0.0090$ ) were independent risk factors associated with mortality in COVID-19 patients. However, diabetes mellitus, liver cirrhosis, and inflammatory bowel disease were not risk factors for mortality.

\section{Conclusion}

Male, elderly patients $₫ 65$ years old, Medical Aid beneficiaries, higher $\mathrm{CCl}(\geq 3)$, and living in a large outbreak area might have a greater risk of mortality and comorbidities such as hypertension, COPD, CHF, and ESRD could also greatly affect the mortality in COVID-19 patients.

\section{Background}

The coronavirus disease 2019 (COVID-19) caused by severe acute respiratory syndrome coronavirus 2 (SARS-CoV-2), first reported in Wuhan, China in December, 2019 has rapidly spread worldwide and has become a public health emergency (1). Since there are no available vaccines and effective treatments, increasing the number of COVID-19 patients and mortality is a major challenge to public health. Compared with severe acute respiratory syndrome (SARS) and Middle East respiratory syndrome (MERS), COVID-19 has a lower mortality among diagnosed COVID-19 patients. However, previous studies have

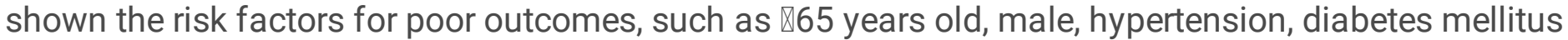
(DM), and cardiovascular diseases (2-4). Lai et al. reported that mortality was correlated with country health care resources (5). In many countries, there were undiagnosed COVID-19 cases by reverse 
transcription polymerase chain reaction (RT-PCR) test and the limitation in availability of health care services, such as lack of intensive care units and ventilators for adequate treatment of severe patients. Republic of Korea (hereafter 'Korea'), like other countries, has had a COVID-19 outbreak since the first patient on January 3,2020 . Social distancing and the use of facial masks have been recommended since the beginning of the COVID-19, and most populations have followed these recommendations well. In addition, early detection through active diagnostic tests, contact tracing, and quarantine were performed during the initial outbreak of COVID-19 (6). If patients were confirmed to have COVID-19, the patients were classified into mild, moderate, severe, and extremely severe cases by the public health center. All patients were admitted to a hospital or Living and Treatment Center for proper management, classified according to severity. Mild cases are isolated at the Living and Treatment Centers and monitored by health care staff at least twice a day. If the patient's symptoms were aggravated, patients were transported to the hospital. When symptoms improved, the patients were discharged based on isolation release standards. That is, the health care services in Korea are maintained, so the degree of mortality and the risk factors related to mortality may be different from those in previous studies. Therefore, we aimed to identify the clinical risk factors for mortality in the COVID-19 patients without health system disruption.

\section{Methods}

\section{Data collection}

The Ministry of Health and Welfare and the Health Insurance Review and Assessment Service (HIRA) of Korea have shared the nationwide data of COVID-19 for global research collaboration on COVID-19. The HIRA dataset was based on insurance benefit claims and comprised all cases confirmed as COVID-19 in Korea. This dataset contains various health-related variables such as socio-demographic information, health care utilization including diagnosis, and survival status. This study was approved by the Institutional Review Board of the National Health Insurance Service of Ilsan Hospital, and the study adhered to the tenets of the Declaration of Helsinki (NHIMC 2020-05-002). The need for written informed consent was waived as the patient identification data were deleted from the database used.

\section{Definition}

Patients with COVID-19 were defined by the following diagnostic codes using the HIRA dataset: corona virus infection (B342); coronavirus as the cause of diseases classified into other chapters (B972); domestic temporary designation or emergency use of new diseases (B18); novel coronavirus infection (U181); coronavirus disease 2019 (U071). All patients with COVID-19 were confirmed by RT-PCR test.

The clinical characteristics of the population were obtained when the COVID-19 diagnosis was confirmed. According to the International Classification of Diseases, $10^{\text {th }}$ Revision (ICD-10), the comorbidities were categorized as respiratory disease (chronic obstructive pulmonary disease [COPD], J43-44; asthma, J4546), heart disease (congestive heart failure, 150; ischemic heart disease, 120-25, except 120.1), hypertension (110), diabetes mellitus (E10-14), and cancer (C00-97). End-stage renal disease (ESRD) was 
defined as patients who submitted claims for any procedure or material associated with either hemodialysis or peritoneal dialysis (special dialysis claim codes V001 and V003; treatment codes of 07020 for hemodialysis and 07061 for peritoneal dialysis) combined with the ICD-10 code for chronic kidney disease and end-stage kidney disease (N18). Liver cirrhosis was defined using ICD-10 codes (K702, K703, K704, K717, K720, K721, K729, K740-K746, K761, K766-K767, R18, 1850, I859, 1864, I868, 1982, and 1983) within liver diseases (K70-K77). Inflammatory bowel disease (IBD), including ulcerative colitis (UC) and Crohn disease (CD), was defined using ICD-10 codes and registration codes from the RID patient support program (CD: K 50 and V130, UC: K51 and V131) (7). The Charlson Comorbidity Index (CCl), which is most widely used for calculating the adjusted risk of mortality based on comorbidities, was calculated as previously described (8).

\section{Statistical analysis}

Descriptive statistics were performed for all variables. Differences between the two groups were assessed using the chi-squared test for categorical variables. Multiple logistic regression analysis was performed to estimate the odds ratio (OR) and $95 \%$ confidence intervals (Cls) for factors that independently related mortality. A $p$-value $<0.05$ was considered statistically significant. All statistical analyses were performed using SAS version 9.4 (SAS Institute, Cary, NC, USA).

\section{Results}

\section{Baseline characteristics of the population tested for COVID-19.}

Between January 3 and June 1, 2020, a total of 7,333 patients were diagnosed with COVID-19. Of these, 2,966 (40.4\%) were male patients (Table 1 ). The median age of the patients was 48.0 years ( $\min , \max ; 19$, 98). The most common age group was $18-35$ years, and $18.3 \%$ of the patients were $₫ 65$ years old. The number of patients living in Daegu and Gyeongsangbuk-do province, which had a large outbreak of COVID-19, was 4,025 (54.9\%), and the number of Medical Aid beneficiaries was 619 (8.4\%). A total of $2,472(33.7 \%)$ patients had $\mathrm{CCl}=0$ and $1,799(24.5 \%)$ had $\mathrm{CCl} \geq 3$. Among the comorbidities, the most common medical history in patients was hypertension (26.0\%), followed by DM (23.0\%) and asthma (21.3\%).

\section{Comparison between survivor and non-survivor}

The comparison of socio-demographic and clinical factors between survivor and non-survivor of patients with COVID-19 is shown in Table 2. In all, 227 (3.1\%) patients died. The non-survivor group showed the following characteristics: older age, higher percentage of males, patients confirmed in Daegu and Gyeongsangbuk-do province, and more Medical Aid beneficiaries than in the negative group $(P<0.001)$. The non-survivor group also had a higher $\mathrm{CCl}$ score compared to the negative group $(\mathrm{P}<0.001)$. All underlying diseases except liver cirrhosis and IBD were more common in the non-survivor group.

\section{Risk factors for mortality of COVID-19}


A multivariate logistic regression analysis was performed to identify the risk factors for mortality in patients with COVID-19 (Table 3). Male (OR $=1.92,95 \% \mathrm{Cl}[1.43-2.59], \mathrm{P}<0.0001)$ and older age $(\mathrm{OR}=$ $11.00,95 \% \mathrm{Cl}[7.16-16.88], \mathrm{P}<0.00010)$ were risk factors related to mortality. Patients confirmed in a large outbreak area (Daegu and Gyeongsangbuk-do province) were associated with an increased OR for mortality $(\mathrm{OR}=2.61,95 \% \mathrm{Cl}[1.73-3.96], P<0.0001)$. Compared with National Health Insurance beneficiaries, Medical Aid beneficiaries remained at increased risk of mortality (OR $=1.54,95 \% \mathrm{Cl}[1.05-$ 2.27], $P<0.0282)$. Higher $\mathrm{CCl}$ scores $(\geq 3)$ were associated with increased odds of mortality $(\mathrm{OR}=3.25$, $95 \% \mathrm{Cl}[1.29-2.80], P=0.0013)$. Comorbidities such as hypertension, COPD, CHF, and ESRD were also significantly associated with increased odds of mortality. (hypertension: $\mathrm{OR}=1.90,95 \% \mathrm{Cl} 1.29-2.80$ ), $P$ $=0.0013$; $\mathrm{COPD}: \mathrm{OR}=1.61,95 \% \mathrm{Cl}[1.03-2.51], P=0.0374 ; \mathrm{CHF}: \mathrm{OR}=2.14,95 \% \mathrm{Cl}[1.45-3.15], P=$ 0.0001; ESRD: OR $=5.69,95 \% \mathrm{Cl}[1.55-20.97), P=0.0090)$. DM, liver cirrhosis, and IBD were not significant risk factors for mortality.

\section{Discussion}

Our study is a nationwide study using the Korean HIRA database to evaluate several risk factors for mortality in adults in patients with COVID-19 in Korea. The mortality rate of patients with COVID-19 was $3.1 \%$. Particularly, male, older age (>65 years), patients diagnosed with a large outbreak area, Medical Aid beneficiaries, higher $\mathrm{CCI}(\geq 3)$, hypertension, COPD, CHF, and ESRD were associated with high odds of mortality.

Previous studies have found that women are less susceptible to viral infection than men (9). Because Xchromosome and sex hormone influence the innate and adaptive immune responses to a pathogen. Simultaneously, men are more likely to have underlying diseases. As a result, the proportion of men with mortality is greater than that of women. Old age was a significant clinical predictor of mortality in SARS and MERS $(10,11)$. This study confirmed that old age was also associated with death in patients with COVID-19. The age-dependent defects in immunity function and the overproduction of type 2 cytokines could cause a deficiency in control of viral replication and prolonged pro-inflammatory responses and more likely to develop to poor outcomes (12). Daegu and Gyeongsangbuk-do province had the first large outbreak of COVID-19 in Korea(13). Korea is one of the countries with large outbreak to flatten the curve of the newly developed COVID-19. Although it has been performed without block strict specific areas and shutting down the economy, during this time, a shortage of inpatient beds, healthcare workers, and personal protective equipment in these areas might cause higher mortality rates than in other parts of Korea. In Korea, the Medical Aid system is a public assistance program that provides healthcare benefits to low-income patients. Medical Aid beneficiaries accounted for $2.9 \%$ of all Korea, whereas other Koreans were beneficiaries of National Health Insurance (14). In Korea, the cost of treatment is free of charge after the patients are diagnosed with COVID-19 regardless of the insurance status. In other words, Medical Aid beneficiaries, even if unrelated to the economic status of receiving treatment, fully implicated a risk factor

of mortality in this study. Several studies reported that Medical Aid beneficiaries were possibly related to household poverty and had poor health status and a higher risk of chronic diseases compared to 
National Health Insurance beneficiaries (15). For these reasons, it is thought that Medical Aid Beneficiaries are a risk factor for the mortality

Circulatory, endocrine, and respiratory comorbidities were common among patients with COVID-19 in this study. Our findings have reflected recently published studies in terms of the similarity of comorbidities in patients with COVID-19(16-18). Consistent with previous reports, the percentage of patients with malignancy, ESRD, and IBD was low. These findings provide further objective evidence, with a larger sample size to consider baseline underlying diseases to evaluate prognosis among patients with COVID19.

Several existing studies have reported risk factors for poor outcomes (age $₫ 65$ years, male, hypertension, DM, COPD, malignancy, smoking, and cardiovascular diseases) among patients with COVID-19 admitted to the hospital (2-4). In our study, some risk factors were the same as those of previous studies, but DM, malignancy, and liver cirrhosis were not risk factors for mortality. Perhaps due to the characteristics of Korean medical services (easy accessibility and National Health Insurance Corporation Checkup), most patients with underlying diseases are diagnosed early and receive treatment; thus, there are many patients with COVID-19 who have less complications or have stable conditions of underlying diseases. In addition, this difference in risk factors is thought to have occurred because all patients diagnosed with COVID-19 were able to receive proper management according to severity free of charge after active rapid testing in Korea.

The clinical significance of our study is as follows. First, since large outbreaks become a risk factor for mortality, it is important to prevent large outbreaks in the area by social distancing, wearing masks, early detection through active diagnostic tests, contact tracing, and quarantine. Second, in the case of lowincome patients, the mortality rate is relatively high, therefore, more active social support and interest are required. Third, in general, comorbidity may not exist alone, but two or more comorbidities may exist simultaneously in a patient. A recent study reported that patients with a greater number of comorbidities correlated with poorer clinical outcomes among patients with COVID-19(4). In this study, it was also confirmed that the mortality was significantly increased in patients with $\mathrm{CCl} \geq 3$.

This study had several limitations. First, all patients did not reach the study outcome or were discharged by the end of the study period. Second, due to the retrospective study design using the Korean HIRA database, all clinical data (symptoms, smoking history, BMI, and laboratory tests) were not available. In addition, the severity of comorbidities at the time of confirmation of COVID-19 could not be evaluated. Third, the diagnosis of comorbidities was based on the ICD-10 code. Therefore, the diagnosis of some comorbidities might not be optimal. Fourth, the Korean health care system was not disrupted, so it is free to use the health care system. ICU and ventilator capacity were not exceeded during the study period. Because the health care system and strategy of COVID-19 in each county are different, the above must be considered when interpreting our findings.

\section{Conclusions}


In conclusion, this study analyzed the risk factors for progression to mortality in COVID-19 patients. More attention to these risk factors and more personalized and specific approach are needed to reduce mortality.

\section{Abbreviations}

SARS-CoV-2: severe acute respiratory syndrome coronavirus 2; SARS: severe acute respiratory syndrome; MERS: Middle East respiratory syndrome; RT-PCR: reverse transcription polymerase chain reaction; HIRA: Health Insurance Review and Assessment Service; COVID-19: Coronavirus Disease 2019; CCI: Charlson Comorbidity Index; DM: diabetes mellitus; COPD: chronic obstructive pulmonary disease; IHD: ischemic heart disease; ESRD: end-stage renal disease; IBD: inflammatory bowel disease; UC: ulcerative colitis; CD: Crohn disease

\section{Declarations}

\section{Acknowledgements}

The authors appreciate healthcare professionals dedicated to treating COVID-19 patients in Korea, the Ministry of Health and Welfare, and the Health Insurance Review \& Assessment Service of Korea for promptly sharing the invaluable national health insurance claims data.

\section{Authors' contributions}

Se Jun Park, Hyungkook Lee: Data curation and writing the original draft.Tae Mi Youk: Data curation and methodology. Han Ho Jeon: Conceptualization, writing (review and editing), and supervision. The authors read and approved the final manuscript.

\section{Funding}

None

\section{Availability of data and materials}

Not applicable

Ethics approval and consent to participate

Not applicable

\section{Consent for publication}

Not applicable

\section{Competing interests}


The authors declare that they have no competing interests.

\section{Author details}

${ }^{1}$ Department of Internal Medicine, National Health Insurance Service Ilsan Hospital, Goyang, Republic of Korea. ${ }^{2}$ Research Institute, National Health Insurance Service Ilsan Hospital, Goyang, Republic of Korea. ${ }^{3}$ Department of Statistics, Korea University, Seoul, Republic of Korea

\section{References}

1. WHO Director-General's opening remarks at the media briefing on COVID-19-7 September 2020. https://www.who.int/dg/speeches/detail/who-director-general-s-opening-remarks-at-the-mediabriefing-on-covid-19--7-september-2020/; 2020, Accessed date: 23 September 2020.

2. Zheng Z, Peng F, Xu B, Zhao J, Liu H, Peng J, et al. Risk factors of critical \& mortal COVID-19 cases: A systematic literature review and meta-analysis. J Infect. 2020;81(2):e16-e25.

3. Zhou F, Yu T, Du R, Fan G, Liu Y, Liu Z, et al. Clinical course and risk factors for mortality of adult inpatients with COVID-19 in Wuhan, China: a retrospective cohort study. Lancet. 2020;395(10229):1054-62.

4. Guan WJ, Liang WH, Zhao Y, Liang HR, Chen ZS, Li YM, et al. Comorbidity and its impact on 1590 patients with COVID-19 in China: a nationwide analysis. Eur Respir J. 2020;55(5).

5. Lai CC, Wang CY, Wang YH, Hsueh SC, Ko WC, Hsueh PR. Global epidemiology of coronavirus disease 2019 (COVID-19): disease incidence, daily cumulative index, mortality, and their association with country healthcare resources and economic status. Int J Antimicrob Agents. 2020;55(4):105946.

6. Korea's Response to COVID-19 and Future Direction 7 May, 2020. http://www.mofa.go.kr/viewer/skin/doc.html? $f n=20200520085445765$.pdf\&rs=/viewer/result/202009/; Accessed date; 23 September 2020.

7. Yang BR, Choi NK, Kim MS, Chun J, Joo SH, Kim H, et al. Prevalence of extraintestinal manifestations in Korean inflammatory bowel disease patients. PLoS One. 2018;13(7):e0200363.

8. Charlson ME, Pompei P, Ales KL, MacKenzie CR. A new method of classifying prognostic comorbidity in longitudinal studies: development and validation. J Chronic Dis. 1987;40(5):373-83.

9. Gal-Oz ST, Maier B, Yoshida H, Seddu K, Elbaz N, Czysz C, et al. ImmGen report: sexual dimorphism in the immune system transcriptome. Nat Commun. 2019;10(1):4295.

10. Choi KW, Chau TN, Tsang O, Tso E, Chiu MC, Tong WL, et al. Outcomes and prognostic factors in 267 patients with severe acute respiratory syndrome in Hong Kong. Ann Intern Med. 2003;139(9):715-23.

11. Hong KH, Choi JP, Hong SH, Lee J, Kwon JS, Kim SM, et al. Predictors of mortality in Middle East respiratory syndrome (MERS). Thorax. 2018;73(3):286-9.

12. Opal SM, Girard TD, Ely EW. The immunopathogenesis of sepsis in elderly patients. Clin Infect Dis. 2005;41 Suppl 7:S504-12. 
13. Choi S, Ki M. Estimating the reproductive number and the outbreak size of COVID-19 in Korea. Epidemiol Health. 2020;42:e2020011.

14. National Health Insurance Serive, Health insurance review \& assessment servive:2019.

15. Bahk J, Kang HY, Khang YH. Trends in life expectancy among medical aid beneficiaries and National Health Insurance beneficiaries in Korea between 2004 and 2017. BMC Public Health. 2019;19(1):1137.

16. Huang C, Wang Y, Li X, Ren L, Zhao J, Hu Y, et al. Clinical features of patients infected with 2019 novel coronavirus in Wuhan, China. Lancet. 2020;395(10223):497-506.

17. Chen N, Zhou M, Dong X, Qu J, Gong F, Han Y, et al. Epidemiological and clinical characteristics of 99 cases of 2019 novel coronavirus pneumonia in Wuhan, China: a descriptive study. Lancet. 2020;395(10223):507-13.

18. Richardson S, Hirsch JS, Narasimhan M, Crawford JM, McGinn T, Davidson KW, et al. Presenting Characteristics, Comorbidities, and Outcomes Among 5700 Patients Hospitalized With COVID-19 in the New York City Area. Jama. 2020;323(20):2052-9.

\section{Tables}

Table 1 Baseline characteristics of patients of COVID-19 


\begin{tabular}{|c|c|}
\hline Variables & Overall $(\mathrm{N}=7,333)$ \\
\hline Age, years, median (min, max) & $48.0(19.98)$ \\
\hline $18<$ and $\leq 35, n \%$ & $2,436(33.2)$ \\
\hline $35<$ and $\leq 50, n \%$ & $1,406(19.2)$ \\
\hline $50<$ and $\leq 65, n \%$ & $2,151(29.3)$ \\
\hline$>65, \mathrm{n} \%$ & $1,340(18.3)$ \\
\hline Male, n (\%) & $2,966(40.4)$ \\
\hline Daegu and Gyeonsanbuk-do province, n (\%) & $4,025(54.9)$ \\
\hline Medical Aid beneficiaries, n (\%) & $619(8.4)$ \\
\hline $\mathrm{CCl}, \mathrm{n}(\%)$ & $2,472(33.7)$ \\
\hline 0 & $1,958(26.7)$ \\
\hline 1 & $1,104(15.1)$ \\
\hline 2 & $1,799(24.5)$ \\
\hline \multicolumn{2}{|l|}{$\geq 3$} \\
\hline Hypertension, n (\%) & $1,910(26.0)$ \\
\hline $\mathrm{DM}, \mathrm{n}(\%)$ & $1,687(23.0)$ \\
\hline Asthma, n (\%) & $1,562(21.3)$ \\
\hline COPD, n (\%) & $222(3.0)$ \\
\hline IHD, n (\%) & $541(7.4)$ \\
\hline Congestive heart failure, $\mathrm{n}(\%)$ & $329(4.5)$ \\
\hline Cancer, n (\%) & $425(5.8)$ \\
\hline Liver cirrhosis, n (\%) & $96(1.3)$ \\
\hline ESRD on dialysis, $\mathrm{n}(\%)$ & $19(0.3)$ \\
\hline IBD, n (\%) & $21(0.3)$ \\
\hline
\end{tabular}

COVID-19 Coronavirus Disease 2019, CCI Charlson Comorbidity Index, DM diabetes mellitus, COPD chronic obstructive pulmonary disease, IHD ischemic heart disease, ESRD end-stage renal disease, IBD inflammatory bowel disease

Table 2 Comparison outcomes by COVID-19 related death 


\begin{tabular}{|c|c|c|c|}
\hline Variables & $\begin{array}{l}\text { Non-survivor } \\
(n=227)\end{array}$ & $\begin{array}{l}\text { Survivor } \\
(\mathrm{n}=7,106)\end{array}$ & $P$ value \\
\hline Age, years, median (min, max) & $80.0(36,98)$ & $48.0(19,98)$ & $<0.0001$ \\
\hline Age, years & $0(0.0)$ & $2,436(34.3)$ & $<0.0001$ \\
\hline \multicolumn{4}{|l|}{$18<$ and $\leq 35, n \%$} \\
\hline $35<$ and $\leq 50, n \%$ & $3(1.3)$ & $1,403(19.7)$ & \\
\hline $50<$ and $\leq 65, \mathrm{n} \%$ & $30(13.2)$ & $2,121(29.8)$ & \\
\hline$>65, \mathrm{n} \%$ & $194(85.5)$ & $1,146(16.1)$ & \\
\hline Male, n (\%) & $121(53.3)$ & $2,845(40.0)$ & $<0.0001$ \\
\hline Daegu and Gyeonsanbuk-do province, $n(\%)$ & $198(87.2)$ & $3,827(53.9)$ & $<0.0001$ \\
\hline Medical Aid beneficiaries, n (\%) & $42(18.5)$ & $577(8.1)$ & $<0.0001$ \\
\hline $\mathrm{CCl}, \mathrm{n}(\%)$ & $9(4.0)$ & $2463(34.7)$ & $<0.0001$ \\
\hline 0 & 18 (7.9) & $1,940(27.3)$ & \\
\hline 1 & $26(11.5)$ & $1,078(15.2)$ & \\
\hline 2 & $174(76.7)$ & $1,625(22.9)$ & \\
\hline \multicolumn{4}{|l|}{$\geq 3$} \\
\hline Hypertension, n (\%) & $181(79.7)$ & $1,729(24.3)$ & $<0.0001$ \\
\hline DM, n (\%) & $145(63.9)$ & $1,542(21.7)$ & $<0.0001$ \\
\hline Asthma, n (\%) & $83(36.6)$ & $1,479(20.8)$ & $<0.0001$ \\
\hline COPD, n (\%) & $40(17.6)$ & $182(2.6)$ & $<0.0001$ \\
\hline IHD, n (\%) & $56(24.7)$ & $485(6.8)$ & $<0.0001$ \\
\hline Congestive heart failure, $\mathrm{n}(\%)$ & $63(27.8)$ & $266(3.7)$ & $<0.0001$ \\
\hline Cancer, n (\%) & $41(18.1)$ & $384(5.4)$ & $<0.0001$ \\
\hline Liver cirrhosis, n (\%) & $6(2.6)$ & $90(1.3)$ & 0.0766 \\
\hline ESRD on dialysis, n (\%) & $4(1.8)$ & $15(0.2)$ & 0.0024 \\
\hline IBD, n (\%) & $1(0.4)$ & $20(0.3)$ & 0.4838 \\
\hline
\end{tabular}

COVID-19 Coronavirus Disease 2019, CC/Charlson Comorbidity Index, DM diabetes mellitus, COPD chronic obstructive pulmonary disease, $I H D$ ischemic heart disease, ESRD end-stage renal disease, IBD inflammatory bowel disease 
Table 3 Association between potential risk factors and COVID-19 related death

\begin{tabular}{|lll|}
\hline & \multicolumn{2}{l}{ Multivariate analysis } \\
\hline Variable & OR $(95 \% \mathrm{Cl})$ & P value \\
\hline Male & $1.92(1.43-2.59)$ & $<0.0001$ \\
\hline Age $>$ 65 years & $11.00(7.16-16.88)$ & $<0.0001$ \\
\hline Daegu and Gyeonsanbuk-do province & $2.61(1.73-3.96)$ & $<0.0001$ \\
\hline Medical Aid beneficiaries & $1.54(1.05-2.27)$ & 0.0282 \\
\hline CCI (ref: CCI = 0) & $1.56(0.68-3.55)$ & 0.2901 \\
$=1$ & $2.14(0.96-4.79)$ & 0.0631 \\
$=2$ & $3.25(1.50-7.03)$ & 0.0029 \\
$\geq 3$ & & \\
\hline Hypertension & $1.90(1.29-2.80)$ & 0.0013 \\
\hline DM & $1.29(0.92-1.80)$ & 0.1448 \\
\hline Asthma & $1.00(0.72-1.39)$ & 0.9901 \\
\hline COPD & $1.61(1.03-2.51)$ & 0.0374 \\
\hline IHD & $0.75(0.51-1.10)$ & 0.1391 \\
\hline Congestive heart failure & $2.14(1.45-3.15)$ & 0.0001 \\
\hline Cancer & $1.22(0.81-1.84)$ & 0.3369 \\
\hline Liver cirrhosis & $0.67(0.27-1.67)$ & 0.3909 \\
\hline ESRD on dialysis & $5.69(1.55-20.97)$ & 0.0090 \\
\hline IBD & $1.79(0.21-15.29)$ & 0.5949 \\
\hline
\end{tabular}

COVID-19 Coronavirus Disease 2019, CCI Charlson Comorbidity Index, DM diabetes mellitus, COPD chronic obstructive pulmonary disease, IHD ischemic heart disease, ESRD end-stage renal disease, IBD inflammatory bowel disease 